\title{
Transesophageal endoscopic ultrasound-guided transcarotid fine needle aspiration of a positron emission tomography (PET)-positive mediastinal lymph node
}

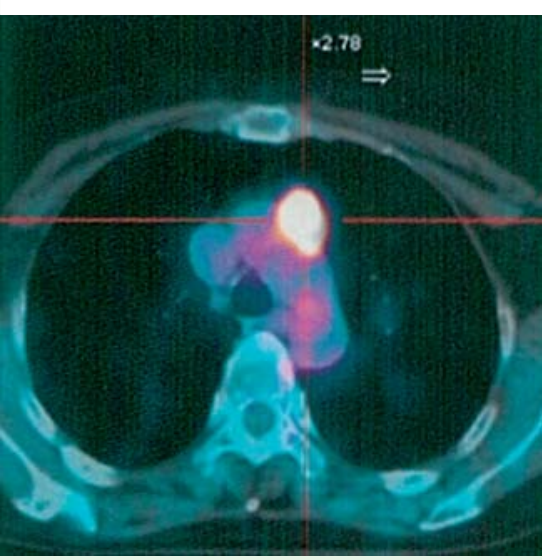

Fig. 1 Image from a positron emission tomography (PET)/computed tomography (CT) scan in a woman with a history of breast cancer showing strong uptake of $18 \mathrm{~F}$-fluorodeoxyglucose (FDG; standardized uptake value [SUV] 7.9) in an enlarged lymph node in the para-aortic region, which was highly suggestive of malignancy.

We report an unusual case of a positron emission tomography (PET)-positive para-aortic lymph node ( $\bullet$ Fig. 1 ) in a patient with breast cancer that was punctured with transesophageal endoscopic ultrasound (EUS)-guided fine needle aspiration (FNA) by traversing the carotid artery, with multiple needle passes performed.

EUS was performed by an experienced endosonographer (A.L.) using a conventional linear echoendoscope. It confirmed the presence of a $15 \times 20-\mathrm{mm}$ lymph node, which was located near to the origin of the left common carotid artery from the aortic arch ( Fig.2). A window that would allow the lymph node to be punctured without traversing any vascular structure could not be found. Therefore, transcarotid EUS-FNA was performed using a 25-gauge needle (Echotip Ultra, Cook Medical Inc., Bloomington, Indiana, USA; $\bullet$ Fig. $3 \mathrm{a}$ and $\odot$ Video 1 ).

After the first needle pass, a hyperechoic halo appeared around the vessel, suggestive of a small leakage of blood, but there were no ultrasonographic signs of overt bleeding ( Fig.3b). Two additional needle passes were performed. The patient was observed for 1 hour in the recovery

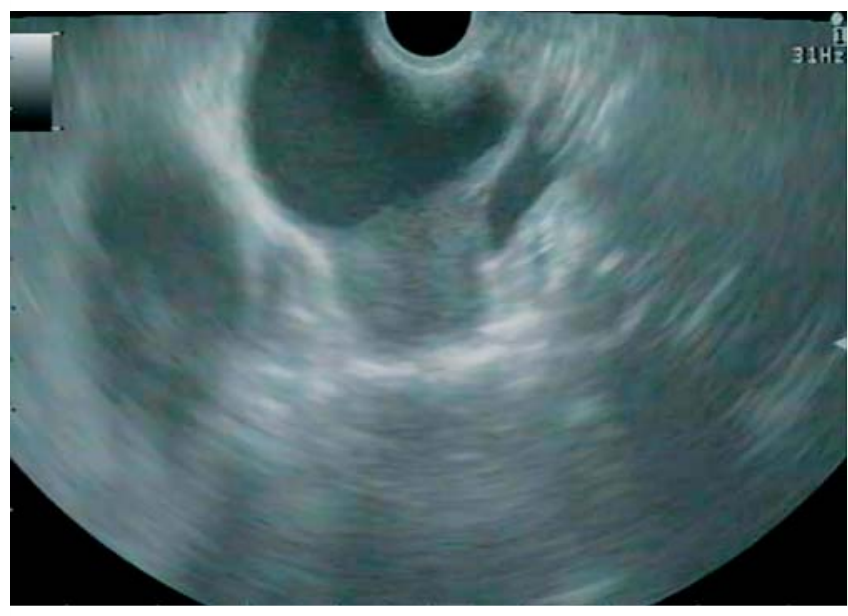

Fig. 2 Transesophageal endoscopic ultrasound (EUS) confirmed the presence of a large $(15 \times 20 \mathrm{~mm})$ mediastinal lymph node located in proximity to the origin of the left common carotid artery from the aortic arch.
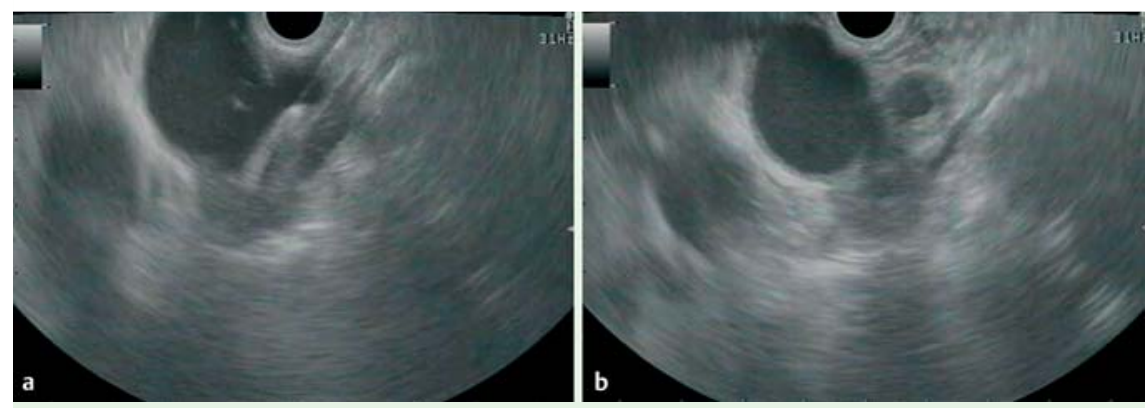

Fig.3 Views during transcarotid endoscopic ultrasound (EUS)-guided fine needle aspiration (FNA) showing: a the enlarged lymph node being punctured with a 25-gauge needle; $\mathbf{b}$ a hyperechoic halo around the vessel after the first needle pass, suggestive of a small leakage of blood, but no ultrasonographic signs of overt bleeding.

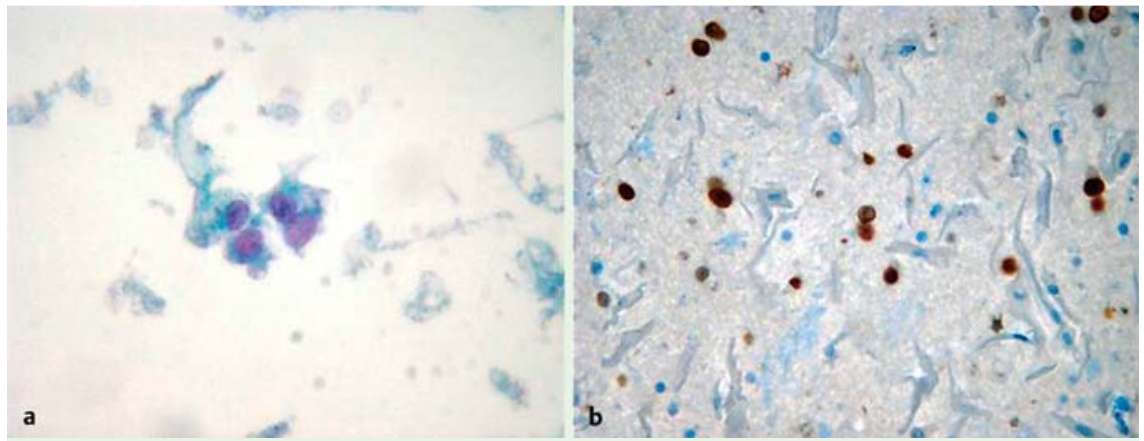

Fig.4 Cytological examination of the aspirated material showing: a small clusters of medium to large cells with pleomorphic nuclei and large amounts of vacuolated cytoplasm suggestive of malignancy; b marked positivity with immunocytochemical staining for estrogen receptors, consistent with a lymph node metastasis from breast cancer.

room then for the following 24 hours with no evidence of complications. She was discharged on the day after the procedure. A definitive diagnosis of metastatic breast cancer was made on cytological examination ( Fig.4a) with evidence of positivity for the estrogen receptor ( Fig.4b).

Recently, Wallace and colleagues [1] have reported the first case of transbronchial endobronchial ultrasound (EBUS)-guided transaortic FNA in a patient with meta- 


\section{Video 1}

A conventional linear echoendoscope was used to confirm the position of the enlarged lymph node in proximity to the origin of the left common carotid artery from the aortic arch. Transcarotid endoscopic ultrasound (EUS)-guided fine needle aspiration (FNA) was performed using a 25-gauge needle. Multiple passes were performed without complications.

static lymph nodes from lung cancer using a 22-gauge needle. Subsequently, von Bartheld [2] utilized the same technique to perform transesophageal transaortic EUS-FNA of para-aortic lymph nodes and lung masses using a 25-gauge needle. In both cases, only a single needle pass was performed because of the fear of complications. In contrast, the present case shows that EUS-FNA of a para-aortic lymph node is also technically feasible by traversing the carotid artery and that no complications resulted even when multiple passes of a 25 -gauge needle were carried out.
Endoscopy_UCTN_Code_TTT_1AS_2AF

Competing interests: None

F. Lococo ${ }^{1}$, F. De Vincentis ${ }^{2}$, A. Naldini ${ }^{3}$, F. Tsiopoulos ${ }^{2}$, A. Paglia ${ }^{3}$, G. Fadda ${ }^{4}$, A. Cesario ${ }^{5}$, P. Granone ${ }^{1}$, G. Costamagna ${ }^{2}$, A. Larghi ${ }^{2}$

${ }^{1}$ Department of Thoracic Surgery, Catholic University, Rome, Italy

2 Digestive Endoscopy Unit, Catholic University, Rome, Italy

${ }^{3}$ Department of Gynecology, Catholic University, Rome, Italy

${ }^{4}$ Department of Pathology, Catholic University, Rome, Italy

${ }^{5}$ IRCCS San Raffaele Pisana, Rome, Italy

\section{References}

1 Wallace MB, Woodward TA, Raimondo $M$ et al. Transaortic fine-needle aspiration of centrally located lung cancer under endoscopic ultrasound guidance: the final frontier. Ann Thorac Surg 2007; 84: 1019-1021

2 von Bartheld MB, Rabe KF, Annema JT. Transaortic EUS-guided FNA in the diagnosis of lung tumors and lymph nodes. Gastrointest Endosc 2009; 69: 345-349

Bibliography

DOI http://dx.doi.org/

10.1055/s-0032-1310060

Endoscopy 2012; 44: E402-E403

(c) Georg Thieme Verlag KG

Stuttgart · New York

ISSN 0013-726X

Corresponding author

\section{F. Lococo, MD}

Department of Thoracic Surgery

Catholic University of Sacred Heart

Largo F. Vito $n$

Roma

Italy

Fax: +39-32-94131202

filippo_lococo@yahoo.it 